\title{
Green and Efficient PEG-Based Ultrasonic-Assisted Extraction of Polysaccharides from Tree Peony Pods and the Evaluation of Their Antioxidant Activity In Vitro
}

\author{
Xiaonan Zhang, Qingfeng Ban $\mathbb{D}^{D}$, Xibo Wang ${ }^{(D)}$, and Zhongjiang Wang $(\mathbb{D}$ \\ Key Laboratory of Soybean Biology in Chinese Ministry of Education, Northeast Agricultural University Harbin 150030, China \\ Correspondence should be addressed to Xibo Wang; wangxibo@neau.edu.cn and Zhongjiang Wang; wzjname2018@163.com
}

Received 29 June 2018; Accepted 20 October 2018; Published 1 November 2018

Academic Editor: Yun-Peng Chao

Copyright (c) 2018 Xiaonan Zhang et al. This is an open access article distributed under the Creative Commons Attribution License, which permits unrestricted use, distribution, and reproduction in any medium, provided the original work is properly cited.

\begin{abstract}
We adopted and developed an ultrasonic-assisted extraction method to obtain polysaccharides from tree peony pods using polyethylene glycol (PEG) as the solvent. The technological parameters have been designed as a single factor to enhance the tree peony pod polysaccharide extraction yield. Specific conditions (ultrasound irradiation power, $250 \mathrm{~W}$; ultrasound irradiation time, $30 \mathrm{~min}$; reaction temperature $50^{\circ} \mathrm{C}$; liquid-solid ratio, $25 \mathrm{~mL} / \mathrm{g}$; and concentration of PEG, $0.2 \mathrm{~g} / \mathrm{mL}$ ) generated an experimental yield of $14.14 \% \pm 0.44 \%$. Subsequently, the monosaccharide composition of the tree peony pod polysaccharides was determined by HPLC using a 1-phenyl-3-methyl-5-pyrazolone precolumn derivatization method. The results indicated that tree peony pod polysaccharides contained mannoses, rhamnose, glucuronic acid, galacturonic acid, glucose, galactose, arabinose, and fucose with a molar ratio of $1.44: 2.87: 0.32: 18.99: 3.99: 10.21: 0.96: 1.85: 0.21$. The tree peony pod polysaccharides obtained are mainly galacturonic acid and galactose, which are acidic polysaccharides. Finally, the antioxidant activities (DPPH and FRAP) of the tree peony pod polysaccharides were assessed, and the compounds exhibited moderate antioxidant activities.
\end{abstract}

\section{Introduction}

Plant polysaccharides are a class of macromolecular compounds that contain more than ten monosaccharides and are polymerized by glycosidic bonds. In recent years, studies have shown the pharmacological effects of polysaccharides, such as antitumor [1,2], anti-inflammatory [3], antioxidation $[4,5]$, antiaging [5], and cardiovascular disease inhibition [6]. Because plant polysaccharides are natural compounds and have a wide range of biological activities, research on polysaccharides has attracted more and more attention. In recent years, research on the separation, purification, chemical structure, and pharmacological activities of polysaccharides has gone viral. Tree peony is a ligneous plant that belongs to the family Ranunculaceae [7]. It is an endemic species in China and exists mainly in central and southwestern China. Peony has more than 2,000 years of cultivation history in China, mainly for ornamental and medicinal use [7]. Peony root skin as a traditional Chinese medicine is included in the Pharmacopoeia of the People's Republic of China (TCM) [7].
Peony seed oil was approved by the Chinese government as a new resource food in 2011 [8]. People realize that the demand for peony seed oil is increasing. The large demand for peony seed oil will result in the production of a large number of peony pods. Therefore, research on the components in the pods has gradually gained attention [9]. Peony pods are approximately $60 \%$ of the fruit's weight. Pods that are discarded after processing are generally planted or used as low-value fuels. People have been paying more and more attention to the comprehensive use of tree peony resources.

Studies have shown that tree peony pods contain luteolin and apigenin [10], as well as polysaccharides [11]. Tree peony polysaccharides have been reported to have pharmacological effects such as antioxidant [11], hypoglycemic [12], immune regulation [13], and hypolipidemic activities [14]. Choosing the right extraction method depends on the characteristics of the plant material, including the complexity of the material, product cost, environmental effects, and safety. Polysaccharides are natural complex supramolecular compounds that are difficult to extract under mild conditions [15]. At present, 
the most common method to extract polysaccharides is hot water extraction. However, the disadvantages of hot water extraction are obvious; the compounds are extracted over a long period of time with high extraction temperatures and a low extraction rate, which may lead to polysaccharide degradation during the extraction process [16]. Ultrasound assisted extraction (UAE) is a new technology that has been developed in recent years and has been successfully applied to the extraction of many plant active ingredients [17]. With the advantages of speeding up the extraction efficiency, saving energy, and environmental protection, it is considered a "Green Technology." UAE has broad application prospects in the extraction of thermally unstable active ingredients and foods that require low temperature processing. With the continuous research and improvements in equipment and technology, UAE will have more extensive application prospects in the fields of food, medicine, and chemical engineering. Ultrasound is a mechanical wave with an effective frequency generally in the range from 20 to $100 \mathrm{kHz}$. UAE extracts cell contents by the application of the combined effect of cavitations, vibration, pulverization, and agitation generated by ultrasonic waves to destroying cell walls. Thus, choosing the best scheme to improve the extraction efficiency of polysaccharides is critical to reduce the time and energy consumption. PEG solution as a green solvent has the advantages of low price, low flammability, nonvolatile, and low-toxicity [18]. Additionally, PEG is relatively stable at high temperatures and acidity, and a water and organic solvent has better solubility in a redox system $[19,20]$. Polyethylene glycol is a polar molecule suitable for ultrasonic energy dissipation [21]. Due to the excellent properties described above, PEG solution is an effective, gentle, and safe extracting solvent compared with traditional and modern solvents. Polyethylene glycol has been used in the extraction of polysaccharides, and its effects are obvious [20,21].

There is little research focused on the extraction of polysaccharides from tree peony pods; therefore, it is necessary to improve the extraction rate of polysaccharides from peony pod. Additionally, further studies on the bioactivity of polysaccharide extractions with PEG as a solvent are still needed. In this study, PEG solution was used as an extracting solvent to extract tree peony pod polysaccharides under ultrasound irradiation. A single factor combined with response surface methodology was used to optimize the extraction parameters such as the PEG molecular weight, PEG concentration, soaking time, liquid-solid ratio, reaction temperature, ultrasound irradiation power, and ultrasound irradiation time. After deproteinization, decolorization, and desalting of the obtained crude polysaccharides, the glycidic configuration was determined. Finally, the antioxidant activity of the obtained pod polysaccharides was compared in vitro.

\section{Materials and Methods}

2.1. Materials and Chemicals. The tree peony fruit was collected in August 2017 from the outskirts of Tongling, Anhui Province, China. After peeling the seeds, they were preserved and dried in a cool and ventilated place. After drying, the preserved pod was crushed with a pulverizer and the 60-80 mesh portion was taken as the experimental material. The moisture content was determined to be $10.2 \%$. The pulverized preserved pod material was stored in a desiccator at a low temperature $\left(4-6^{\circ} \mathrm{C}\right)$ and protected from light until it was extracted.

Tripyridyltriazine (TPTZ), 1,1-diphenylpicrylcarbonyl radical (DPPH), 1-phenyl-3-methyl-5-pyrazolone (PMP), and DEAE-cellulose and L-ascorbic acid (Vitamin C, VC) were purchased from Sigma-Aldrich (St. Louis, MO, USA). Polyethylene glycol at molecular weights of 200, 400, 600, 800, and 1000 and papain (E.C. 3.4.22.2; $800 \mathrm{U} / \mathrm{mg}$ ) was purchased from Shanghai Yuanye Bio-Technology Co Ltd. (Shanghai, China). The monosaccharide D-glucose, D-galactose, D-glucuronic acid, D-galacturonic acid, Dmannose, L-xylose, L-arabinose, L-rhamnose, and L-fucose reference substances were purchased from Shanghai Aladdin Biochemical Technology Co., Ltd. (Shanghai, China). AB-8 macroporous resin (particle diameter $0.3-1.2 \mathrm{~mm}$, surface area $650-700 \mathrm{~m} 2 / \mathrm{g}$, average pore diameter $8.5-9 \mathrm{~nm}$, and cross-linked polystyrene) was purchased from Cangzhou Bon Adsorber Technology Co., Ltd. (Cangzhou, Heibei, China). To eliminate the monomers and porogenic agents from the holes in the macroporous resin during the synthesis procedure, a preliminary adsorbent bead treatment process was performed as follows: the resin was immersed in ethanol solution $(95 \%, \mathrm{v} / \mathrm{v})$ for $24 \mathrm{~h}$ at $25^{\circ} \mathrm{C}$, washed thoroughly with deionized water until no residue ethanol was present, and finally dried under vacuum prior to use. Ultrapure water used in the solutions and dilutions was obtained from a Milli-Q purification system (Bedford, MA, USA).

2.2. Experimental Apparatus. The experimental ultrasonic bath KQ250DB (Kunshan, Jiangsu, China) was used as an extraction device (250 W maximum output power and 40 $\mathrm{kHz}$ irradiation frequency). The rectangular bath has a size of $500 \times 300 \times 150 \mathrm{~mm}$. It can be continuously adjusted through power feedback/control depending on the actual needs for the ultrasound irradiation, ranging from $100 \mathrm{~W}$ to $250 \mathrm{~W}$, and maintained through a connection to a constant temperature water bath.

2.3. Polysaccharide Extraction Procedure. First, 10.0 g (dry) of accurately preserved fruit was placed it in a $250-\mathrm{mL}$ Erlenmeyer flask. It was extracting in the ultrasonic bath under various conditions to determine the range for the extraction factors and the appropriate values for each factor. Each extraction was filtered, and the filtrate was combined with absolute ethanol to reach an ethanol concentration of $80 \%$. The suspension was stored overnight in a refrigerator at $4^{\circ} \mathrm{C}$, after which the floccule precipitates were isolated by centrifugation $\left(8000 \times \mathrm{g}\right.$ for $30 \mathrm{~min}$, at $\left.20^{\circ} \mathrm{C}\right)$, washed with absolute ethanol three times, and ultimately freeze-dried. The polysaccharide yield was calculated gravimetrically. Each experiment was conducted in triplicate to ensure the accuracy of the experimental results.

2.4. Decolorization, Deproteinization, and Desalination. The obtained crude polysaccharides contain many impurities, 
mainly pigment and protein. Thus, after $2 \mathrm{~h}$ of filtering in decoloring solution, we dissolved $5 \mathrm{~g}$ of crude polysaccharides in $500 \mathrm{~mL}$ ultra-pure water and added $\mathrm{AB}-8$ resin to adjust the solution to $4 \mathrm{pH}$. We adjusted the $\mathrm{pH}$ of the filtrate to 7 using sodium hydroxide solution. The Papain-Sevage method was used to deproteinize the tree peony pod crude polysaccharides based on the procedure by Wang et al. [22] with slight modifications. Briefly, a $10 \mathrm{mg} / \mathrm{mL}$ polysaccharide solution was prepared. Then, $50 \mathrm{~mL}$ of papain with a polysaccharide mass of $3 \%$ was added and the solution was shaken in a water bath at $60^{\circ} \mathrm{C}$ for $4 \mathrm{~h}$. After enzymolysis, the papain was inactivated at $100^{\circ} \mathrm{C}$ for $5 \mathrm{~min}$, cooled, and centrifuged at $5000 \times \mathrm{g}$ for $15 \mathrm{~min}$ and, then, the supernatant was collected. $\mathrm{N}$-butanol and chloroform were mixed in a 1:4 ratio $(\mathrm{v} / \mathrm{v})$ to prepare the Sevage reagent, and an appropriate amount was mixed with the supernatant obtained above. After shaking for $20 \mathrm{~min}$, the solution was centrifuged at $3000 \times \mathrm{g}$ for $5 \mathrm{~min}$; this was repeated twice. Four volumes of absolute ethanol were added to supernatant, and the mixture was stirred and alcoholized overnight; finally, the polysaccharide was washed with absolute ethanol and acetone and freeze-dried.

Desalination was performed by dialysis. Deionized water was used to prepare a polysaccharide sample solution with a concentration of approximately $10 \mathrm{mg} / \mathrm{mL}$. This sample solution was solubilized by ultrasound and filtered to remove insoluble impurities before the filtrate was loaded into a dialysis bag. Both ends of the dialysis bag were clamped and magnetic dialysis was performed in deionized water for $48 \mathrm{~h}$. The water was changed every 3 hours. After the polysaccharide solution was removed from the dialysis bag, 4 volumes of absolute ethanol were added to it, followed by stirring and drying overnight, filtering, and freeze-drying.

2.5. General Analysis of Purified Polysaccharides. The purified polysaccharides were collected for further analysis. The total sugar content was determined by the phenol-sulfuric acid method using glucose as the reference substance [23]. The polysaccharide monosaccharide composition analysis was performed as described by Honda et al. [24]. The monosaccharide composition of the purified polysaccharides was analyzed according to a previously reported method [25].

2.6. Determination of DPPH Free Radical Scavenging Capacity. As per a previously described method [26] with slight modifications, $0.1 \mathrm{~mL}$ of the $95 \%$ ethanol volume fraction from different concentrations of tree peony pod polysaccharides was added to $3.9 \mathrm{~mL}$ of $25 \mathrm{mg} / \mathrm{L} \mathrm{DPPH}$ ethanol solution. After incubation for $30 \mathrm{~min}$ in the dark, the absorbance was measured at $517 \mathrm{~nm}$ and counted as the Asample. The absorbance determined by replacing the sample with the same volume of the $95 \%$ ethanol volume fraction is the Acontrol. The DPPH free radical scavenging ability was expressed as SC\% using the following equation: SC\% $=(1$ - Asample/Acontrol) $\times 100 \%$, where the Acontrol does not contain sample. The absorbance value of the solution at $t=$ 0 and all the other absorbance values were measured 3 times. The results were averaged, and a nonlinear regression curve was fit to obtain the IC50 value.

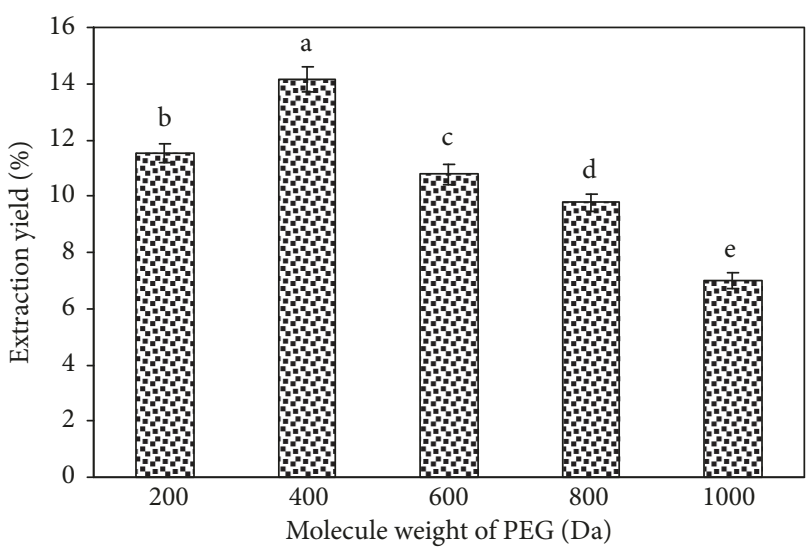

FIgURE 1: Effects of molecule weight of PEG. Values are mean \pm SD ( $n=3$ replicates). Means that have different letters at the top of each bar are significantly different $(\mathrm{P}<0.05)$.

\subsection{Determination of Ferric-Reducing Antioxidant Power} (FRAP). The FRAP was determined according to a previously described method [27] with some modifications. First, $25 \mathrm{~mL}$ of $300 \mathrm{mM}$ acetate buffer ( $\mathrm{pH}$ 3.6) was mixed with $2.5 \mathrm{~mL}$ of $10 \mathrm{mM}$ TPTZ solutions and $2.5 \mathrm{~mL}$ of $20 \mathrm{mM}$ ferric chloride solution. The mixture was maintained at $37^{\circ} \mathrm{C}$ for $30 \mathrm{~min}$ and used as the FRAP solution, Next, $150 \mu \mathrm{L}$ of tree peony pod polysaccharide sample was added to $2850 \mu \mathrm{L}$ FRAP solution, which was then incubated in the dark for 30 $\min$. The absorbance was measured at $593 \mathrm{~nm}$, and the result was expressed as the $\mu \mathrm{mol}$ per gram of Trolox (TE)/g.

2.8. Statistical Analysis. All experiments were replicated three times. Each replicate is expressed as mean $\pm S D$, and an ANOVA test (using SPSS 17.0) was used to compare the mean values of each treatment. Significant differences between the means of parameters were determined using Duncan's test $(\mathrm{p}<0.05)$.

\section{Results and Discussion}

\subsection{Optimization the Extraction Conditions of Polysaccharides}

3.1.1. The Influence of PEG Molecular Weight and Concentration on Peony Pod Polysaccharide Yield. The polarity of watersoluble polyethylene glycol solution depends on the weight of the polyethylene glycol molecule during the extraction process. We examined the molecular weight of polyethylene glycol, and the experimental results are shown in Figure 1. Considering the extraction rate, PEG400 had advantages over the other PEG molecular weights. This illustrates that PEG400 is similar to polar solutions in the literature [20,28]. Therefore, PEG400 was suitable as the extraction solvent. The polyethylene glycol concentration significantly influences the polarity and viscosity of polyethylene glycol aqueous solutions. From Figure 2, the extraction rate increased when the PEG concentration was $0-0.20 \mathrm{~g} / \mathrm{mL}$ and decreased when the PEG concentration was $0.20-0.50 \mathrm{~g} / \mathrm{mL}$, which is similar to the extraction of polysaccharides from Nelumbo nucifera 


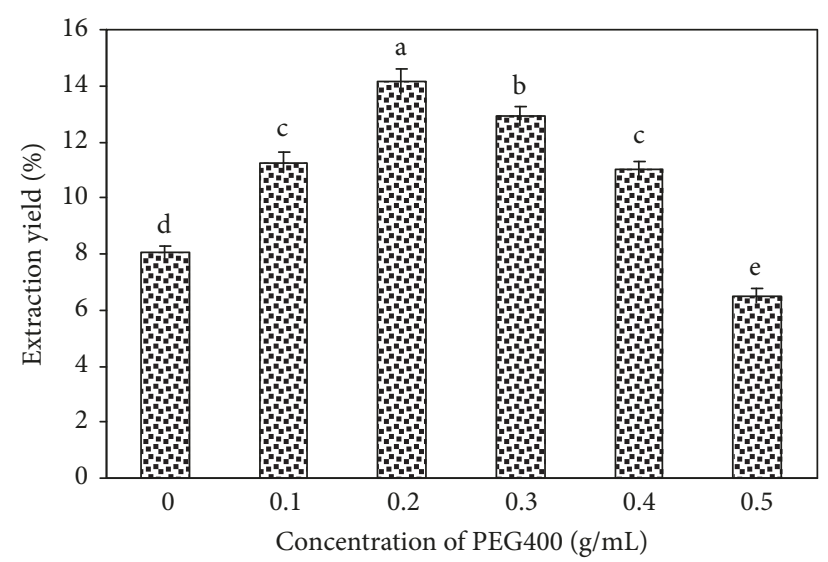

Figure 2: Effects of concentration of PEG400 solution. Values are mean $\pm S D$ ( $n=3$ replicates). Means that have different letters at the top of each bar are significantly different $(\mathrm{P}<0.05)$.

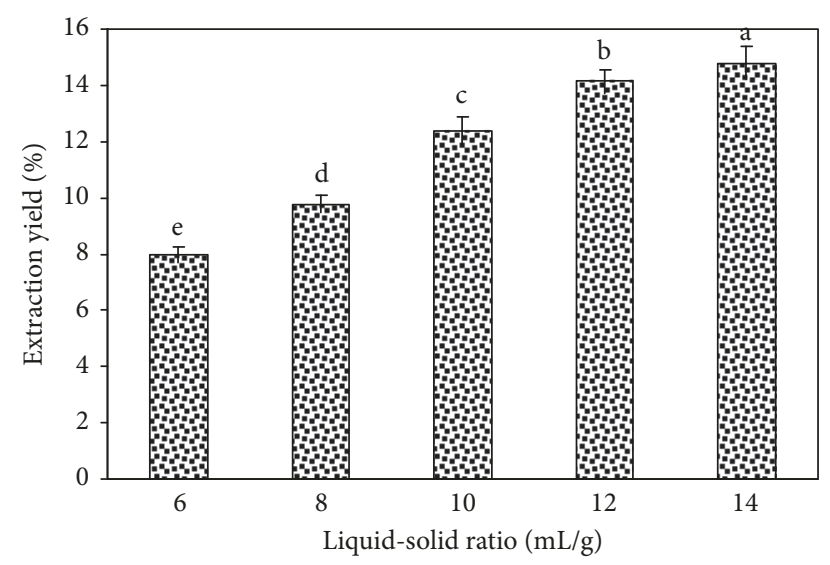

FIGURE 3: Effect of liquid-solid ratio on the extraction yield of tree peony pod polysaccharides. Values are mean $\pm S D(n=3$ replicates). Means that have different letters at the top of each bar are significantly different $(\mathrm{P}<0.05)$.

[28]. Thus, $0.20 \mathrm{~g} / \mathrm{mL}$ PEG400 was adopted for use in this study.

3.1.2. Effect of the Liquid-Solid Ratio on the Tree Peony Pod Polysaccharide Yield. The liquid-solid ratio is another important factor that may affect the yield coefficient of the target product. The effect of this parameter on the tree peony pod polysaccharide yield is shown in Figure 3. We can see that increasing the liquid-solid ratio significantly increased the yield of tree peony pod polysaccharides. Increasing the liquid-solid ratio to $12 \mathrm{~mL} / \mathrm{g}$ had few effects on the tree peony pod polysaccharide yield coefficient. At this point, the tree peony pod polysaccharide yield stabilized. Thus, a liquidsolid ratio of $12 \mathrm{~mL} / \mathrm{g}$ was selected as the extraction condition for the tree peony pod polysaccharides.

3.1.3. Effect of Reaction Temperature on the Tree Peony Pod Polysaccharide Yield. Ultrasound temperature is also an important factor affecting the yield of target plant analytes. As

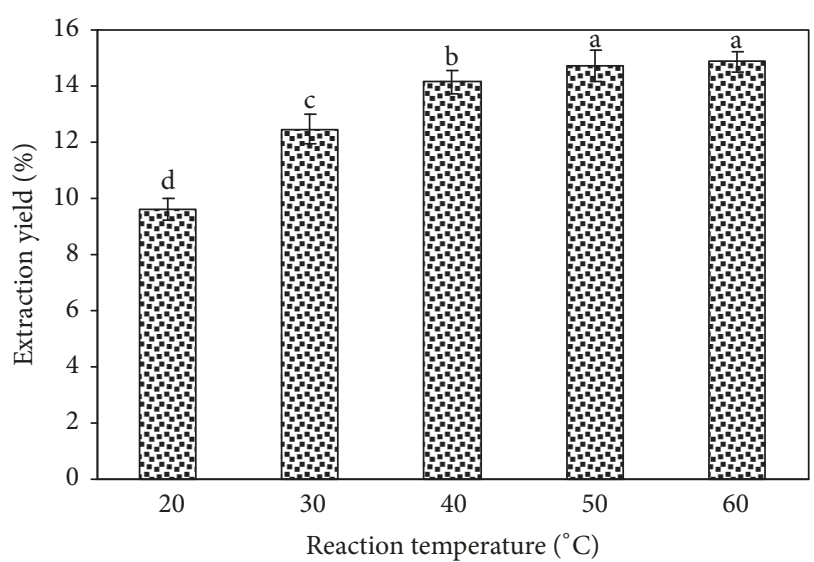

FIGURE 4: Effect of reaction temperature on the extraction yield of tree peony pod polysaccharides. Values are mean \pm SD $(n=3$ replicates). Means that have different letters at the top of each bar are significantly different $(\mathrm{P}<0.05)$.

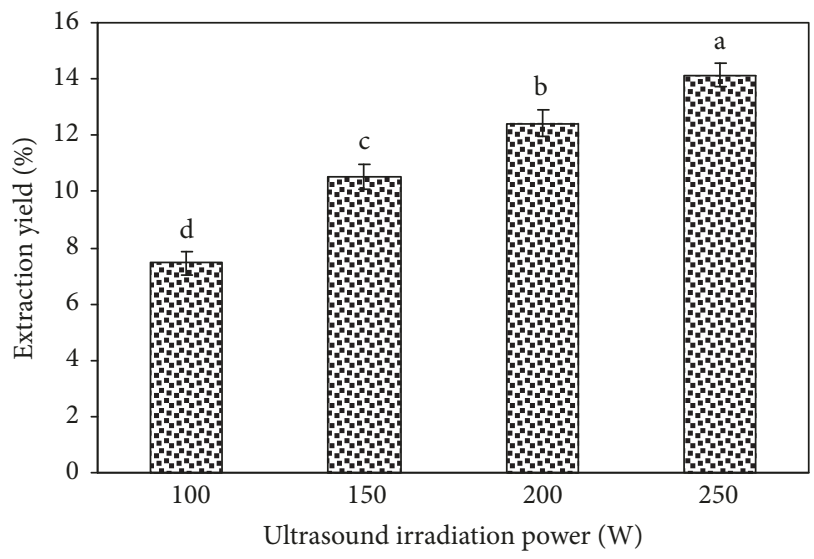

FIGURE 5: Effect of ultrasound irradiation power on the extraction yield of tree peony pod polysaccharides. Values are mean \pm SD $(n=$ 3 replicates). Means that have different letters at the top of each bar are significantly different $(\mathrm{P}<0.05)$.

shown in Figure 4, when the reaction temperature is lower than $20^{\circ} \mathrm{C}$, the tree peony pod polysaccharide yield is less than $9.61 \% \pm 0.39 \%$. The tree peony pod polysaccharide yield is positively correlated with the increase in temperature. For example, as the temperature reached $50^{\circ} \mathrm{C}$, the tree peony pod polysaccharide yield reached $14.14 \% \pm 0.44 \%$. Continuously increasing the reaction temperature led to a slow increase in the yield of the tree peony pod polysaccharide. Because the higher temperature is not conducive to the stability of the tree peony pod polysaccharides results, $50^{\circ} \mathrm{C}$ was selected as the best extraction temperature for the ultrasonic extraction of polysaccharides.

3.1.4. Effect of Ultrasound Irradiation Power on the Tree Peony Pod Polysaccharide Yield. The effect of ultrasound irradiation power on the tree peony pod polysaccharide yield is shown in Figure 5. We can see from the figure that when other extraction factors are fixed, the polysaccharide yield 


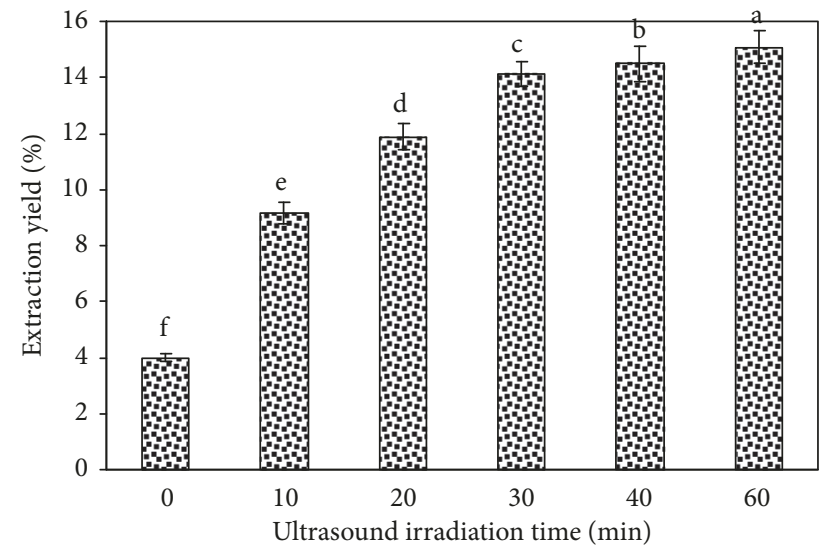

FIGURE 6: Effect of ultrasound irradiation time on the extraction yield of tree peony pod polysaccharides. Values are mean \pm SD (n $=3$ replicates). Means that have different letters at the top of each bar are significantly different $(\mathrm{P}<0.05)$.

increases with increasing ultrasound irradiation power. The use of ultrasound mechanical cavitation and thermal effects can increase the media molecule movement speed, thereby increasing the penetration into the media and improving the yield of the active ingredient from the raw material. When the ultrasound irradiation power reached $250 \mathrm{~W}$, the polysaccharide yield was the highest $(\mathrm{p}<0.05)$; thus, 250 $\mathrm{W}$ was the best irradiation power for the ultrasound-assisted extraction of tree peony pod polysaccharides.

3.1.5. Effect of Ultrasound Irradiation Time on the Tree Peony Pod Polysaccharide Yield. The effect of different ultrasound irradiation times on the tree peony pod polysaccharide yield is shown in Figure 6. From the figure, as the ultrasonic irradiation time increases, the tree peony pod polysaccharide yield also increases. When the ultrasound irradiation time is $30 \mathrm{~min}$, the increase due to the time shows limited improvement in the tree peony pod polysaccharide yield, and the tree peony pod polysaccharide yield only slightly increases. Therefore, $30 \mathrm{~min}$ is the best ultrasound irradiation time for the ultrasound-assisted extraction.

3.2. Analysis of the Monosaccharide Composition from Tree Peony Pod Polysaccharides. Under the selected conditions, PMP ramification monosaccharides are isolated from the tree peony pod polysaccharide sample was separated from the baseline. The chromatography peaks were identified based on the retention times in the on-line UV spectrogram, indicating that tree peony pod polysaccharides are composed of mannoses, rhamnose, glucuronic acid, galacturonic acid, glucose, galactose, arabinose, and fucose with a molar ratio of $1.44: 2.87: 0.32: 18.99: 3.99: 10.21: 0.96: 1.85: 0.21$. The results show that the obtained tree peony pod polysaccharides are mainly galacturonic acid and galactose, which are acidic polysaccharides.

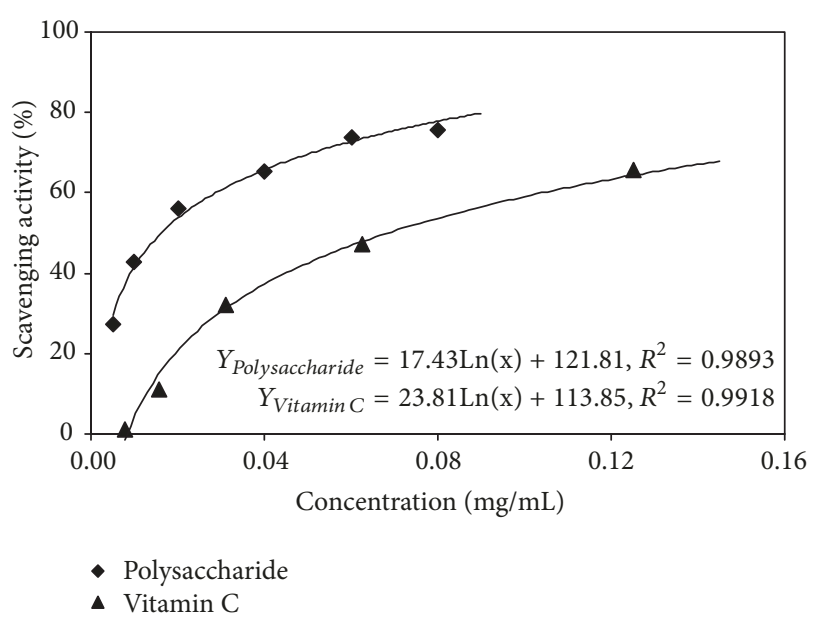

FIGURE 7: Evaluation of DPPH radical-scavenging activity of tree peony pod polysaccharides.

\subsection{In Vitro Antioxidant Activity}

3.3.1. DPPH Free Radical Scavenging Capacity. DPPH reagent was purple-red in methanol and had the strongest absorption at a wavelength of $517 \mathrm{~nm}$. DPPH is structurally stable and can combine with antioxidant hydrogen atoms and electrons and fade. Evaluation of the antioxidant DPPH scavenging capacity was quantitatively tested according to the degree of the color change by the purple-red solution caused by DPPH supplied by antioxidants. The concentration of DPPH is proportional to the absorbance of the solution. The higher the absorbance, the weaker the ability of the antioxidant to scavenge $\mathrm{DPPH}$, and the weaker the antioxidant capacity of the tested substance. The DPPH scavenging capacity of tree peony pod polysaccharides and vitamin $\mathrm{C}$ is shown in Figure 7 . When the vitamin $C$ concentration is the same as the tree peony pod polysaccharides, the DPPH scavenging ability of the tree peony pod polysaccharides is significantly higher than that of vitamin C. Increasing the tree peony pod polysaccharides concentration increased their scavenging capacity. When the polysaccharide concentration was 0.08 $\mathrm{mg} / \mathrm{mL}$, the DPPH clearance rate of the tree peony pod polysaccharides was $75.5 \%$. In contrast, when the vitamin C concentration reached $0.125 \mathrm{mg} / \mathrm{mL}$, its DPPH scavenging capacity was only $65.5 \%$. Therefore, the tree peony pod polysaccharides had a strong scavenging ability for DPPH.

3.3.2. Ferric-Reducing Antioxidant Power (FRAP). $\mathrm{Fe}^{3+}$ in the tripyridyltriazine (TPTZ) reagent can be reduced to $\mathrm{Fe}^{2+}$ by antioxidants; the solution is blue, and the absorbance is maximum at a wavelength of $593 \mathrm{~nm}$. Figure 8 showed that tree peony pod polysaccharide FRAP is weaker than that of vitamin C. Increasing the polysaccharide concentration gradually increased tree peony pod polysaccharide FRAP; thus, the antioxidant activity of the tree peony pod polysaccharides was positively correlated with its concentration. 


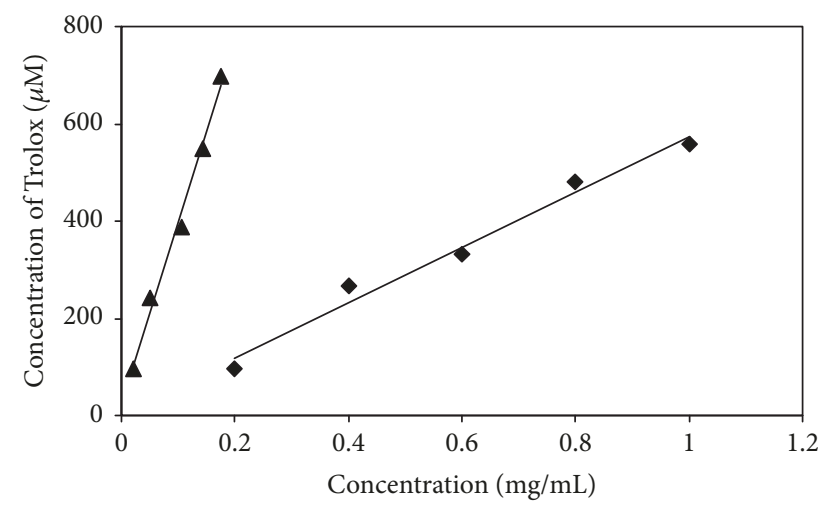

- Polysaccharide

$\Delta$ Vitamin C

FIgURE 8: Evaluation of ferric reducing antioxidant power of tree peony pod polysaccharides.

\section{Conclusions}

In this paper, tree peony pod was used as a raw material with PEG400 solution as an extraction solvent for the ultrasoundassisted extraction of polysaccharides. We optimized the single-factor test extraction conditions for tree peony pod polysaccharides (PEG400 dose of $0.2 \mathrm{~g} / \mathrm{mL}$, liquid-solid ratio $25 \mathrm{~mL} / \mathrm{g}$, reaction temperature $50^{\circ} \mathrm{C}$, ultrasound irradiation power $250 \mathrm{~W}$, and ultrasound irradiation time $30 \mathrm{~min}$ ). Under these conditions, the polysaccharide yield from tree peony pods was $14.14 \% \pm 0.44 \%$. The tree peony pod polysaccharides are composed of mannoses, rhamnose, glucuronic acid, galacturonic acid, glucose, galactose, arabinose, and fucose with a molar ratio of $1.44: 2.87: 0.32: 18.99: 3.99: 10.21$ : $0.96: 1.85: 0.21$. The tree peony pod polysaccharides are acid polysaccharides that are mainly composed of galacturonic acid and galactose. The results from the analysis and evaluation of their antioxidant activities showed that the DPPH free radical scavenging capacity of tree peony pod polysaccharides was significantly higher than that of vitamin $\mathrm{C}$ at the same concentration. The FRAP of tree peony pod polysaccharides was lower than that of vitamin C. However, the FRAP of tree peony pod polysaccharides is positively correlated with its concentration.

\section{Data Availability}

The data used to support the findings of this study are included within the article.

\section{Conflicts of Interest}

The authors declare no conflicts of interest.

\section{Acknowledgments}

The authors acknowledge the financial support from the NNSF of China (number 31701568), Key Research and Development Projects of Shandong Province (number 2018YYSP021), Natural Science Foundation of Heilongjiang
Province (number C2018025), Young Innovative Talent Training Plan of Heilongjiang Province (number UNPYSCT2016143), Young Talents Project of Northeast Agricultural University (number 17QC16), and Postdoctoral Start-Funded Research of Heilongjiang Province (number LBH-Q17027).

\section{References}

[1] Y. Masuda, K. Ito, M. Konishi, and H. Nanba, "A polysaccharide extracted from Grifola frondosa enhances the antitumor activity of bone marrow-derived dendritic cell-based immunotherapy against murine colon cancer," Cancer Immunology, Immunotherapy, vol. 59, no. 10, pp. 1531-1541, 2010.

[2] W. Wang, M. Liu, Y. Wang et al., "Lycium barbarum Polysaccharide Promotes Maturation of Dendritic Cell via Notch Signaling and Strengthens Dendritic Cell Mediated T Lymphocyte Cytotoxicity on Colon Cancer Cell CT26-WT,' Evidence-Based Complementary and Alternative Medicine, vol. 2018, Article ID 2305683, 2018.

[3] P. Wang, X.-T. Li, L. Sun, and L. Shen, "Anti-inflammatory activity of water-soluble polysaccharide of Agaricus blazei murill on ovariectomized osteopenic rats," Evidence-Based Complementary and Alternative Medicine, vol. 2013, Article ID 164817, 2013.

[4] S. Lin, L. Wen, B. Yang et al., "Improved growth of lactobacillus bulgaricus and streptococcus thermophilus as well as increased antioxidant activity by biotransforming litchi pericarp polysaccharide with aspergillus awamori," BioMed Research International, vol. 2013, Article ID 413793, 2013.

[5] S. Li, H. Liu, W. Wang et al., "Antioxidant and anti-aging effects of acidic-extractable polysaccharides by Agaricus bisporus," International Journal of Biological Macromolecules, vol. 106, pp. 1297-1306, 2018.

[6] S. Benni, T. Avramoglou, H. Hlawaty, and L. Mora, "Dynamic Contact Angle Analysis of Protein Adsorption on Polysaccharide Multilayer's Films for Biomaterial Reendothelialization," BioMed Research International, vol. 2014, Article ID 679031, 2014.

[7] F. Chen, K. Mo, Q. Zhang, S. Fei, Y. Zu, and L. Yang, "A novel approach for distillation of paeonol and simultaneous extraction of paeoniflorin by microwave irradiation using an ionic liquid solution as the reaction medium," Separation and Purification Technology, vol. 183, pp. 73-82, 2017.

[8] F. Chen, X. Zhang, Q. Zhang et al., "Simultaneous synergistic microwave-ultrasonic extraction and hydrolysis for preparation of trans-resveratrol in tree peony seed oil-extracted residues using imidazolium-based ionic liquid," Industrial Crops and Products, vol. 94, pp. 266-280, 2016.

[9] F. Chen, X. Zhang, X. Du, L. Yang, Y. Zu, and F. Yang, "A new approach for obtaining trans-resveratrol from tree peony seed oil extracted residues using ionic liquid-based enzymatic hydrolysis in situ extraction," Separation and Purification Technology, vol. 170, pp. 294-305, 2016.

[10] H. Wang, L. Yang, Y. Zu, and X. Zhao, "Microwave-assisted simultaneous extraction of luteolin and apigenin from tree peony pod and evaluation of its antioxidant activity," The Scientific World Journal, vol. 2014, Article ID 506971, 2014.

[11] H. Wang, Y. Li, W. Liu, H. Wang, Y. Zu, and X. Zhao, "Optimizing polysaccharide extraction from peony tree pod using response surface method and assessing its antioxidative activity," Bulletin of Botanical Research, vol. 35, no. 1, pp. 127132,2015 . 
[12] Q. Wang, C. Liu, Z. Zhao, Y. Xu, and G. Chen, "Study of screening and effects of effective components on hypoglycemic activity of polysaccharide from Mudan cortex," China Journal of Basic Medicine in Traditional Chinese Medicine, vol. 7, no. 5, pp. 18-21, 2001.

[13] L. Dai, Z. Zhao, Y. Shen, and L. Guo, "Effects of polysaccharide of Mudan cortex PSM_2b on immune functions of mice in vitro," Acta Laser Biology Sinica, vol. 10, no. 1, pp. 48-50, 2001.

[14] G. Zhao, Y. Shen, Z. Wei, and C. Ding, "Experimental study of polysaccharides-2b from Mudan cortex of Paeoma suffruticosa Andr on blood rheology of rats with diabetes," Chinese Traditional Patent Medicine, vol. 30, no. 9, pp. 1270-1273, 2008.

[15] Y. Zhang, H. Wang, P. Wang, C. Ma, G. He, and M. R. T. Rahman, "Optimization of PEG-based extraction of polysaccharides from Dendrobium nobile Lindl. and bioactivity study," International Journal of Biological Macromolecules, vol. 92, pp. 1057-1066, 2016.

[16] Z. Hromádková, A. Ebringerová, and P. Valachovič, "Ultrasound-assisted extraction of water-soluble polysaccharides from the roots of valerian (Valeriana officinalis L.)," Ultrasonics Sonochemistry, vol. 9, no. 1, pp. 37-44, 2002.

[17] F. Chemat, N. Rombaut, A. Sicaire, A. Meullemiestre, A. Fabiano-Tixier, and M. Abert-Vian, "Ultrasound assisted extraction of food and natural products. Mechanisms, techniques, combinations, protocols and applications. A review," Ultrasonics Sonochemistry, vol. 34, pp. 540-560, 2017.

[18] L. Liu, R.-L. Liu, J. Zhang, and Z.-Q. Zhang, "Study on the PEGbased microwave-assisted extraction of flavonoid compounds from persimmon leaves," Journal of Separation Science, vol. 35, no. 23, pp. 3412-3420, 2012.

[19] T. Zhou, X. Xiao, G. Li, and Z.-W. Cai, "Study of polyethylene glycol as a green solvent in the microwave-assisted extraction of flavone and coumarin compounds from medicinal plants," Journal of Chromatography A, vol. 1218, no. 23, pp. 3608-3615, 2011.

[20] L. Zhang, Z. Cheng, Q. Zhao, and M. Wang, "Green and efficient PEG-based ultrasound-assisted extraction of polysaccharides from superfine ground lotus plumule to investigate their antioxidant activities," Industrial Crops and Products, vol. 109, pp. 320326, 2017.

[21] L. Zhang, S. Guo, M. Wang, and L. He, "PEG-based ultrasoundassisted enzymatic extraction of polysaccharides from Ginkgo biloba leaves," International Journal of Biological Macromolecules, vol. 80, pp. 644-650, 2015.

[22] X. Wang, Y. Zhang, Z. Liu, M. Zhao, and P. Liu, "Purification, characterization, and antioxidant activity of polysaccharides isolated from cortex periplocae," Molecules, vol. 22, no. 11, Article ID E1866, 2017.

[23] M. Dubois, K. A. Gilles, J. K. Hamilton, P. A. Rebers, and F. Smith, "Colorimetric method for determination of sugars and related substances," Analytical Chemistry, vol. 28, no. 3, pp. 350356, 1956.

[24] S. Honda, E. Akao, S. Suzuki, M. Okuda, K. Kakehi, and J. Nakamura, "High-performance liquid chromatography of reducing carbohydrates as strongly ultraviolet-absorbing and electrochemically sensitive 1-phenyl-3-methyl5-pyrazolone derivatives," Analytical Biochemistry, vol. 180, no. 2, pp. 351-357, 1989.

[25] H. Wang, Y. Li, Z. Ren et al., "Optimization of the microwaveassisted enzymatic extraction of Rosa roxburghii Tratt. polysaccharides using response surface methodology and its antioxidant and $\alpha$-D-glucosidase inhibitory activity," International Journal of Biological Macromolecules, vol. 112, pp. 473-482, 2018.
[26] K. Hou, X. Yang, M. Bao, F. Chen, H. Tian, and L. Yang, "Composition, characteristics and antioxidant activities of fruit oils from Idesia polycarpa using homogenate-circulating ultrasound-assisted aqueous enzymatic extraction," Industrial Crops and Products, vol. 117, pp. 205-215, 2018.

[27] Z. Liu and L. Yang, "Antisolvent precipitation for the preparation of high polymeric procyanidin nanoparticles under ultrasonication and evaluation of their antioxidant activity in vitro," Ultrasonics Sonochemistry, vol. 43, pp. 208-218, 2018.

[28] A. Ahmad, K. M. Alkharfy, T. A. Wani, and M. Raish, "Application of Box-Behnken design for ultrasonic-assisted extraction of polysaccharides from Paeonia emodi," International Journal of Biological Macromolecules, vol. 72, pp. 990-997, 2015. 


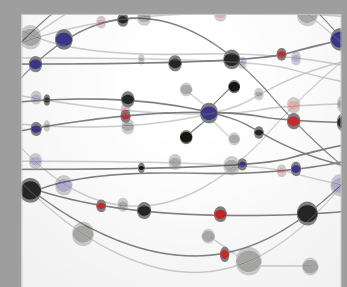

The Scientific World Journal
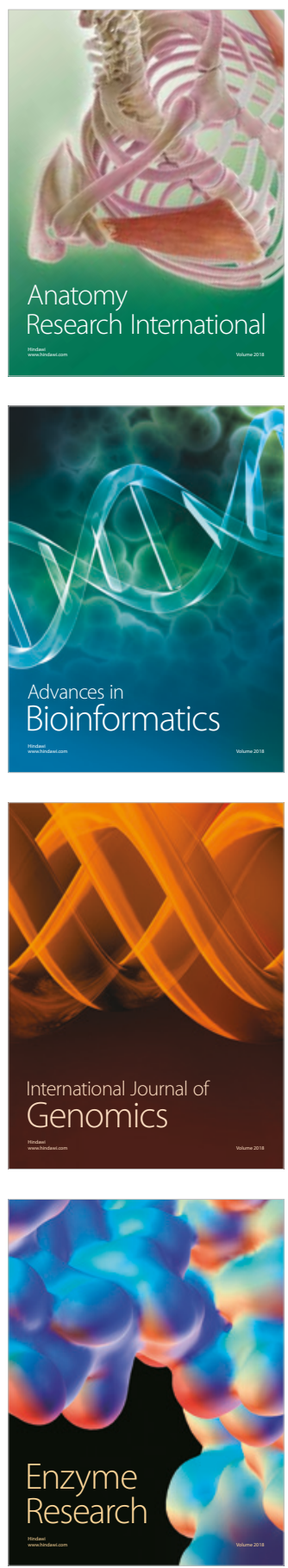
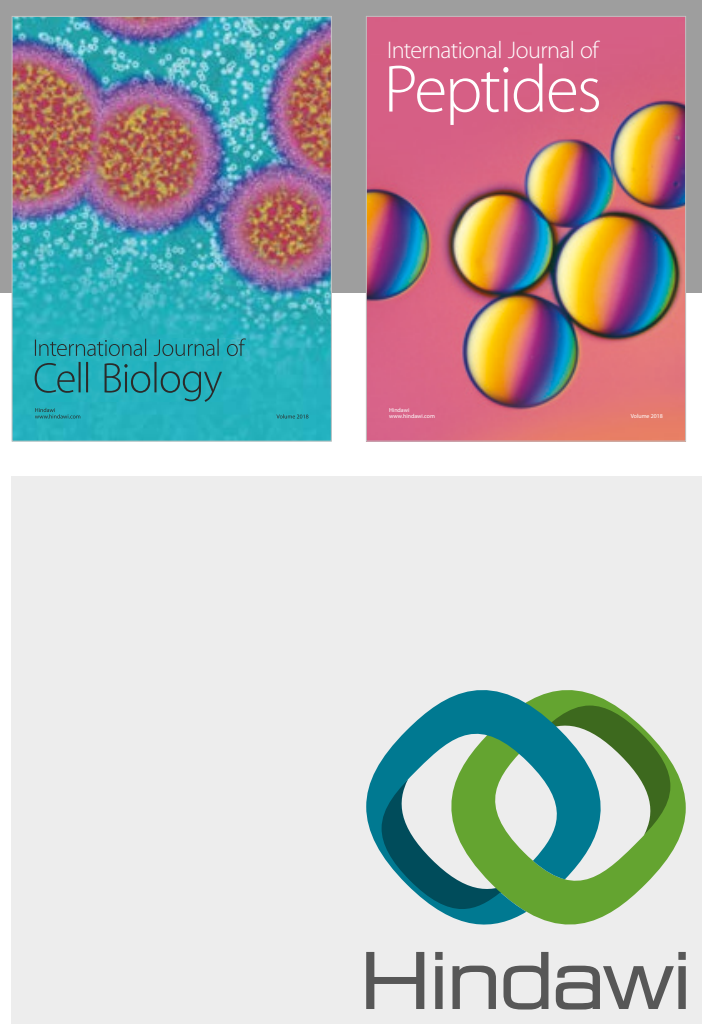

Submit your manuscripts at

www.hindawi.com
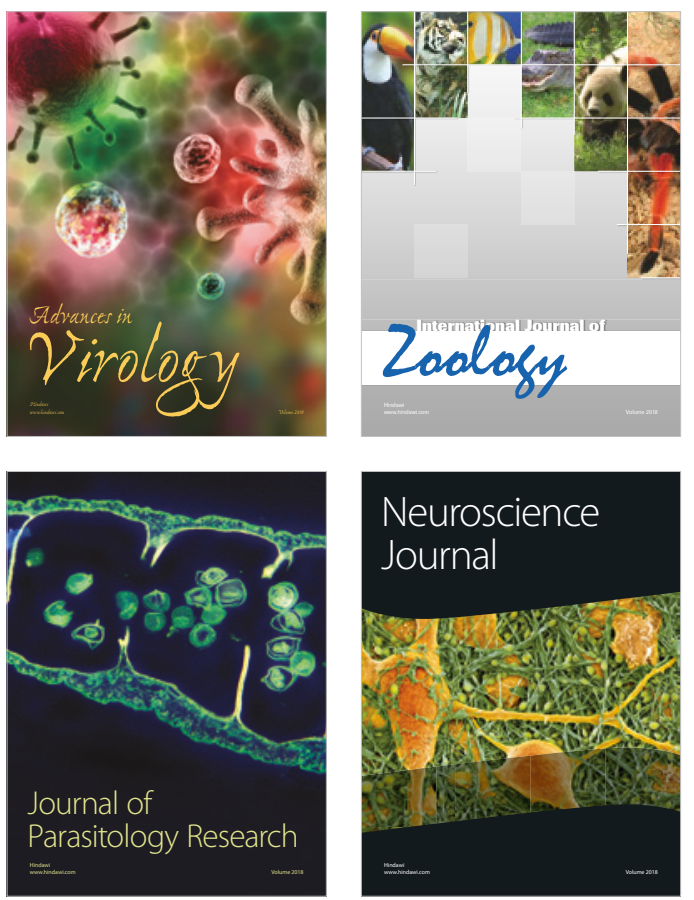
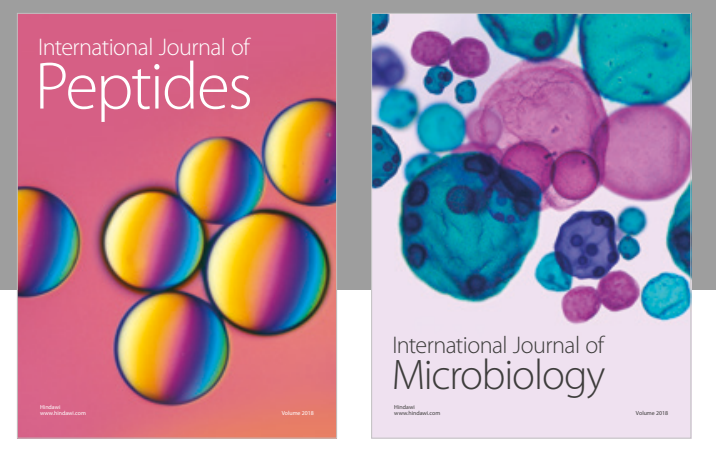

nternational Journal of Microbiology
Journal of
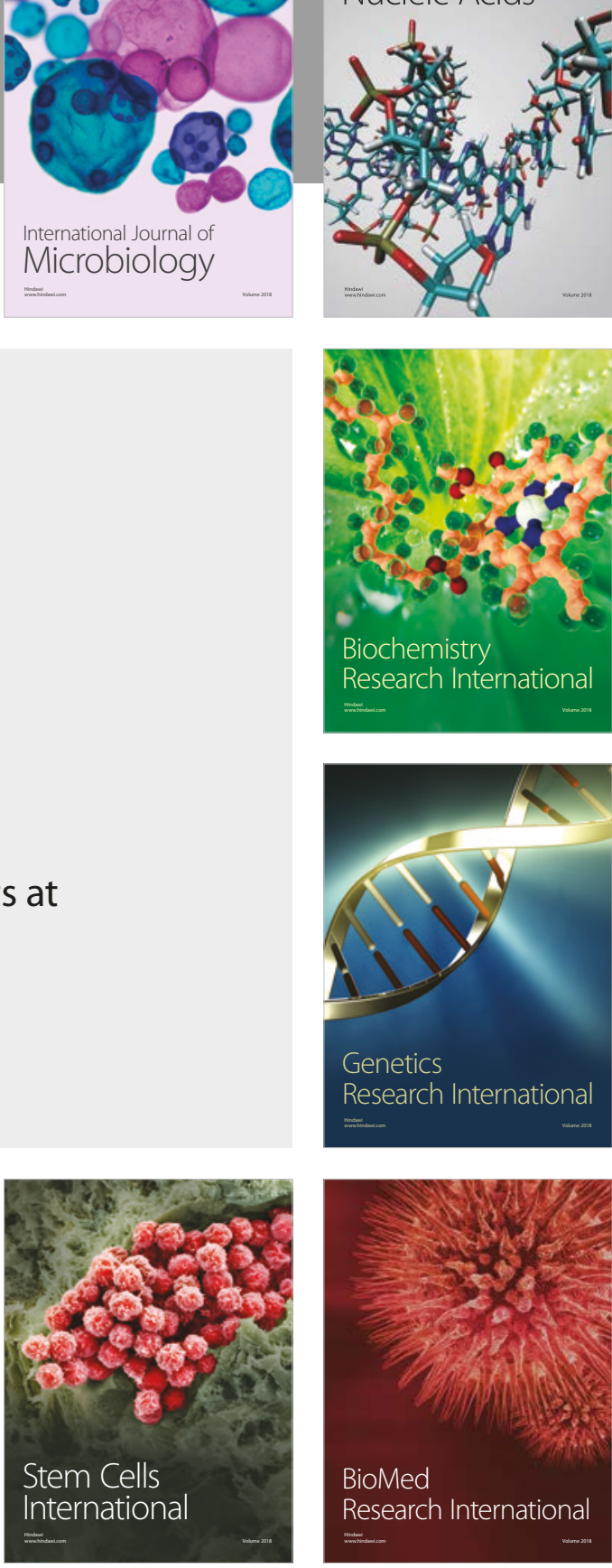
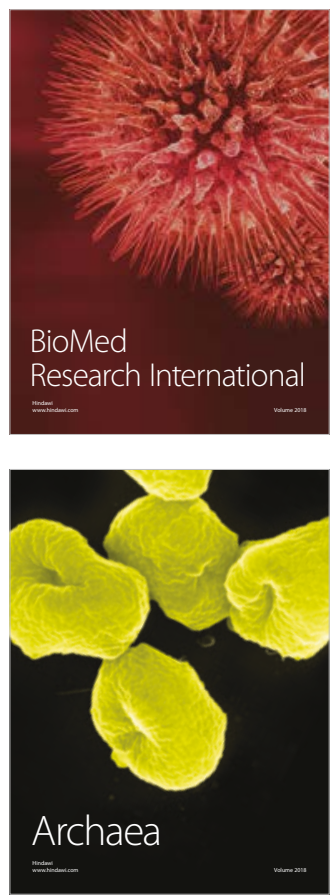